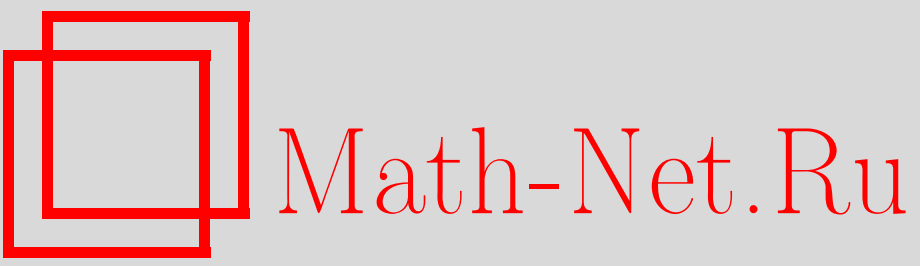

М. В. Комарова, М. Ю. Налимов, Асимптотика старших порядков теории возмущений: первая поправка к константам ренормировки $O(n)$-симметричной теории в $(4-\epsilon)$-разложении, ТMФ, 2005, том 143, номер 2, 211-230

DOI: https://doi.org/10.4213/tmf1811

Использование Общероссийского математического портала Math-Net.Ru подразумевает, что вы прочитали и согласны с пользовательским соглашением http://www.mathnet.ru/rus/agreement

Параметры загрузки:

IP: 54.157 .27 .8

26 апреля 2023 г., $18: 19: 33$ 
ТЕОРЕТИЧЕСКАЯ

И МАТЕМАТИЧЕСКАЯ

ФИЗИКА

Том 143, № 2

май, 2005

(C) 2005 г.

М.В. Комарова*, М. Ю. Налимов*

\section{АСИМПТОТИКА СТАРШИХ ПОРЯДКОВ ТЕОРИИ ВОЗМУЩЕНИЙ: ПЕРВАЯ ПОПРАВКА К КОНСТАНТАМ РЕНОРМИРОВКИ $O(n)$-СИММЕТРИЧНОЙ ТЕОРИИ В $(4-\epsilon)$-РАЗЛОЖЕНИИ}

Исследованы высокие порядки теории возмущений $O(n)$-симметричной $\phi^{4}(4-\epsilon)$ модели в схеме минимальных вычитаний. С учетом специфики $(4-\epsilon)$-схемы минимальных вычитаний вычислены поправки к асимптотической формуле для коэффициентов разложения константы ренормировки $Z_{g}$ и критического индекса $\eta$. Полученные поправки существенно улучшают асимптотическое описание результатов петлевых расчетов.

Ключевые слова: асимптотика высоких порядков, инстантон, $\phi^{4}$-модель, константы ренормировки, схема минимальных вычитаний, $(4-\epsilon)$-разложение.

\section{1. ВВЕДЕНИЕ}

Исследование высоких порядков теории возмущений (ТВ) является важной задачей при рассмотрении полевых моделей. В его основе лежит подход, разработанный в работе [1] и называемый инстантонным, состоящий в обобшении для функционального интеграла метода перевала. В связи с тем, что получаемые ряды оказываются расходящимися, необходимо некоторым образом пересуммировать полученный ответ. При этом результат процедуры пересуммирования сушественно зависит как от значений начальных коэффициентов ряда, вычисленных в рамках ТВ, так и от результатов инстантонного анализа. Результаты работы [1] были применены в физике критических явлений при исследовании и пересуммировании рядов для критических индексов [2]. В работах [1], [2] использовалась $\Lambda$-регуляризация и так называемая "единичная" схема ренормировки (ренормировка в симметричной точке).

Позже в [3] был предложен способ вычисления асимптотик разложения критических индексов $\varphi^{4}$-модели на основе инстантонного анализа ультрафиолетовых (УФ) расходи-

${ }^{*}$ Санкт-Петербургский государственный университет, Санкт-Петербург, Россия. E-mail: mikhail.nalimov@pobox.spbu.ru 
мостей, т.е. фактически способ вычисления констант ренормировки в высоких порядках ТВ. При этом использовалась схема минимальных вычитаний (MB) в $(4-\epsilon)$-разложении как наиболее продвинутая в техническом отношении (вычислено пять порядков ТВ); УФ-расходимости проявлялись в виде полюсов по $\epsilon$. Было показано, что для корректного использования инстантонного подхода в теории $\phi^{4}$-модели в $(4-\epsilon)$-схеме МВ необходимо аккуратное рассмотрение порядка предельных переходов $N, 1 / \epsilon \rightarrow \infty(N-$ порядок ТВ). Возникающие в данной схеме особенности приводят к отличиям от канонической техники [2] при вычислении амплитуды асимптотики высоких порядков для констант ренормировки и критических индексов.

Для обсуждения принципиального вопроса о точности результатов, получаемых методами пересуммирования [2], существенна скорость, с которой петлевые разложения ТВ приближаются к асимптотической формуле. Анализ этого вопроса для $(4-\epsilon)$-схемы MB, проведенный в работе [3], показал, что константы ренормировки монотонно приближаются к своей асимптотике, но достаточно далеки от нее (так, например, для константы ренормировки $Z_{g}$ при $N=5$ наблюдается отличие примерно в семьдесят пять раз). Этот факт ставит под сомнение точность результатов борелевского пересуммирования в данной схеме и вынуждает нас либо продолжать вычисление петлевых разложений, пока они не приблизятся в должной степени к асимптотике, либо интересоваться поправкой порядка $1 / N$ к асимптотической формуле [3].

В работе [4] была рассмотрена поправка к асимптотической формуле Липатова [1], улучшаюшая выход на асимптотику петлевых разложений в единичной схеме ренормировки. Однако, чтобы получить такую поправку для констант ренормировки в $(4-\epsilon)$-схеме МВ, необходимо, как и в [3], провести дополнительные вычисления, связанные с выделением УФ-расходимостей в $N$-м порядке ТВ.

Константа ренормировки $Z_{g}$ (и связанная с ней ренормгрупповая функция $\beta(g)$ ) является главной характеристикой при определении асимптотического поведения критических индексов [3]. Именно она определяет скорость выхода на асимптотику петлевых результатов, поэтому мы ограничились лишь исследованием асимптотики высоких порядков константы ренормировки $Z_{g}$.

В данной работе мы, следуя разработанной в работе [3] идеологии, получим поправку к асимптотике высоких порядков для константы ренормировки $Z_{g}$ и критического индекса $\eta$ в $(4-\epsilon)$-схеме МВ. Будет показано, что конкуренция параметров $1 / N$ и $\epsilon$ приводит к тому, что в отличие от результатов работы [4] вклад в асимптотику высоких порядков вносят поправки по $\epsilon$ к инстантону.

Следует упомянуть еше одну предполагаемую область применения полученных результатов. В [5] был предложен модифицированный метод исследования $\varphi^{4}$-модели, приводящий к принципиально сходящимся рядам ТВ (с конечным радиусом сходимости). Разработанный затем в [6], этот метод приспособлен к $(4-\epsilon)$-схеме МВ. Результат работы [3] для главного члена асимптотики высоких порядков констант ренормировки был использован в [7] для улучшения сходимости рядов модели, рассмотренной в работах [5], [6]. Результат данной работы также может быть использован для дальнейшего улучшения сходимости рядов упомянутого метода. 
Так как данная работа является естественным продолжением работы [3], мы будем согласовывать обозначения с обозначениями работы [3] и, опуская некоторые технические аспекты, подробно описанные при вычислении главного порядка разложения по $1 / N$, ссылаться на соответствующие формулы из [3] в виде (I. номер формулы в [3]).

В разделе 2 коротко изложены основные положения липатовского подхода к вычислению асимптотик высоких порядков разложения по константе связи для частично ренормированной корреляционной функции. В разделе 3 обсуждаются особенности формализма $\epsilon$-разложения. В разделе 4 описывается метод вычисления интеграла по флуктуациям в $(4-\epsilon)$-схеме МВ, а затем, в разделе 5 , вычисляется поправка по $\epsilon$ к этому интегралу (в гауссовом приближении по флуктуациям). В разделе 6 обсуждается техника расчета вычета в точке $\epsilon=0$ для частично ренормированной четыреххвостой корреляционной функции (в асимптотике больших $N$ в главном и поправочном порядках разложения по $1 / N)$. В разделе 7 мы вычисляем поправку к липатовской асимптотике для константы ренормировки $Z_{g}$ и критического индекса $\eta$. В заключении мы анализируем полученные результаты.

\section{2. ОСНОВНЫЕ ПОЛОЖЕНИЯ ЛИПАТОВСКОГО ПОДХОДА}

Напомним коротко ход вычислений и основные формулы работ [1], [3], используемые при расчете липатовских асимптотик для констант ренормировки и критических индексов. Рассматривается модель с действием

$$
S_{R}(\vec{\phi}, g)=\frac{1}{2} Z_{\phi}^{2} \partial_{i} \vec{\phi}_{\alpha} \partial_{i} \vec{\phi}_{\alpha}+\frac{1}{4 !} Z_{\phi}^{4} Z_{g} g \mu^{\epsilon}\left(\vec{\phi}^{2}\right)^{2}
$$

в пространстве размерности $D=4-\epsilon$. Здесь $\vec{\phi}$ - $n$-компонентное векторное поле, символ стоит над векторами в изотопическом $n$-мерном пространстве (в отличие от векторов в $D$-мерном пространстве, над которым задано поле $\vec{\phi}$ и элементы которого выделены жирным шриффтом). Свертки по повторяющимся векторным индексам и интегрирования по координатам здесь и в дальнейшем подразумеваются, $Z_{i} \equiv Z_{i}(g)$ - константы ренормировки $(i=g, \varphi), \mu$ - ренормировочная масса.

Для вычисления коэффициентов $G_{k}^{(N)}$ разложения $k$-хвостой функции Грина $G_{k}(g)$ по константе связи $g$ в $N$-м порядке ТВ (для больших $N$ ) используется введенное в [1] интегральное представление для $G_{k}^{(N)}$, в основе которого лежит известная формула Коши, и исследуемое затем методом перевала. Как было замечено еше в [1], [8], вариационные уравнения, соответствующие точке перевала, не зависят от контрчленной части действия и имеют вид

$$
\left.\frac{\delta J}{\delta \vec{\phi}_{\alpha}}\right|_{Z_{i} \equiv 1}=0,\left.\quad \frac{\partial J}{\partial g}\right|_{Z_{i} \equiv 1}=0, \quad J(\vec{\phi}, g)=S_{R}(\vec{\phi}, g)+N \ln (-g) \text {. }
$$

Решение этих уравнений при $\epsilon=0$ содержит зависимость от произвольных параметров $x_{0}, y$ и единичного вектора $\vec{u}$ :

$$
\vec{\phi}\left(\mathbf{x}, \mathbf{x}_{0}, y, \vec{u}\right)=\frac{\vec{u}}{y^{(D-2) / 2}}\left|\vec{\phi}\left(\frac{\mathbf{x}-\mathbf{x}_{0}}{y}, 0,1, \vec{u}\right)\right| .
$$


Параметры эти отражают, соответственно, трансляционную, масштабную и вращательную инвариантности модели. Методом Фаддеева-Попова по этим параметрам явно выделяются интегрирования, при этом значения самих параметров в точке перевала фиксируются $\left(\mathbf{x}_{0}=0, y=1\right)$ с помощью функции $I$, предложенной Липатовым [1],

$$
\begin{aligned}
I= & \left(-\frac{\bar{g}}{4 !} \int d^{D} \mathbf{x} \bar{\phi}^{4}(\mathbf{x})\right)^{D+1} \delta^{D}\left(-\frac{\bar{g}}{4 !} \int d^{D} \mathbf{x} \bar{\phi}^{4}(\mathbf{x}) \mathbf{x}^{\mu}\right) \times \\
& \times \delta\left(-\frac{\bar{g}}{4 !} \int d^{D} \mathbf{x} \bar{\phi}^{4}(\mathbf{x}) \ln \left(\mathbf{x}^{2}\right)\right) \delta^{n}\left(\overrightarrow{\mathbf{u}}-\frac{\int d^{D} \mathbf{x} \bar{\phi}_{i}(\mathbf{x})|\bar{\phi}|^{l}(\mathbf{x})}{\sqrt{\sum_{i}\left(\int d^{D} \mathbf{x} \bar{\phi}_{i}|\bar{\phi}|^{l}\right)^{2}}}\right) .
\end{aligned}
$$

Для того чтобы решение методом перевала сушествовало и в пространстве размерности $4-\epsilon$, необходимо, прежде чем варьировать функционал $J$, наложить дополнительное условие, фиксирующее масштабную инвариантность модели. Для этого используется одна из дельта-функций в $I$, и в результате появляется дополнительное слагаемое с множителем Лагранжа в первом из вариационных уравнений (2) [8]:

$$
-\Delta^{(D)} \vec{\phi}_{\alpha}+\frac{g \mu^{\epsilon}}{3 !} \vec{\phi}^{2} \vec{\phi}_{\alpha}+4 \rho \vec{\phi}^{2} \vec{\phi}_{\alpha} \ln \left(\mathbf{x}^{2}\right)=0
$$

Здесь $\rho$-множитель Лагранжа, $\Delta^{(D)}$ - оператор Лапласа в $D$-мерном $\mathbf{x}$-пространстве. Второе из вариационных уравнений (2) остается при этом неизменным и может быть записано в виде

$$
\frac{g \mu^{\epsilon}}{4 !} \int d \mathbf{x}\left(\vec{\phi}^{2}(\mathbf{x})\right)^{2}=-\mathbf{N} .
$$

Сферически симметричное решение уравнений (4), (5) - инстантон $g_{\mathrm{st}}, \vec{\phi}_{\mathrm{st}}(\mathbf{x})-$ сушествует и может быть найдено аналитически в виде рядов по $\epsilon$. Для нахождения множителя Лагранжа легко модифицировать способ, описанный в [9]: уравнение (4) домножается на $\vec{\phi}_{\mathrm{st}}(x)$, интегрируется по $\mathbf{x}$ и рассматривается совместно с уравнением, отражающим специфику преобразования варьируемого функционала $J$ при растяжении координат $x \mapsto \lambda x[10]$ :

$$
\left.\frac{\partial J\left\{\vec{\phi}_{\mathrm{st}}(\lambda \mathbf{x})\right\}}{\partial \lambda}\right|_{\lambda=1}=\int d^{D} \mathbf{x}\left((D-2) \frac{\partial \vec{\phi}_{\mathrm{st}} \partial \vec{\phi}_{\mathrm{st}}}{2}+D \frac{g_{\mathrm{st}} \mu^{\epsilon}\left(\vec{\phi}_{\mathrm{st}}^{2}\right)^{2}}{4 !}+2 \rho\left(\vec{\phi}_{\mathrm{st}}^{2}\right)^{2}\right)=0 .
$$

Указанные уравнения совместны, если $\rho=-g_{\mathrm{st}} \epsilon / 48$.

В ходе реализации метода Фаддеева-Попова производятся масштабные растяжения поля и координат, что приводит к замене параметров $\mu$ и $\rho$ на эффективные: $\mu \mapsto \mu y$, $\rho \mapsto \rho(\mu y)^{\epsilon}$. Как и в [3], чтобы указьвать в последующих выражениях зависимость от $N, y, \mu$ явно, перейдем к переменным

$$
\bar{\phi}=|\vec{\phi}| / \sqrt{N}, \quad \bar{g}=N g(\mu y)^{\epsilon}, \quad \bar{\rho}=\rho(\mu y)^{\epsilon} N
$$

Для вычисления констант ренормировки в $N$-м порядке разложения по $g$ рассматриваются функции Грина с $R^{\prime}$-операцией, т.е. с вычитаниями расходимостей подграфов с 
меньшим числом петель. Эта частичная ренормировка осуществляется константами $Z_{i}$ в действии (1), вычисленными в теории возмущений по $g$ вплоть до порядка, учитьвающего контрчлены $g^{K}, K<N$. В размерной регуляризации константы $Z_{i}$ содержат полюсы по $\epsilon$, которые и устраняют все УФ-расходимости неренормированного объекта, кроме поверхностных [11].

Для коэффициента при $g^{N}$ разложения частично ренормированной функции Грина $(N \rightarrow \infty)$ после перехода в импульсное представление и интегрирования по $\vec{u}, x_{0}$ имеem [3]:

$$
\begin{aligned}
G_{k}^{(N)}= & (-1)^{N} N^{N} N^{(k+n+3) / 2} \int_{0}^{\infty} d y y^{k-5+\epsilon(k / 2-1)} \times \\
& \times \exp \left(-N J\left(\bar{\phi}_{\mathrm{st}}, \bar{g}_{\mathrm{st}}(\mu y)^{-\epsilon}\right)\right) \gamma_{k}\left(\mathbf{p}_{1}, \mathbf{p}_{2}, \ldots, \mathbf{p}_{k}, y, \epsilon\right),
\end{aligned}
$$

где $\gamma_{k}$ - множитель, содержащий интеграл по флуктуациям вокруг инстантона. Интеграл в данном выражении требует специального исследования, так как именно в нем проявляются поверхностные расходимости (в виде полюсов по $\epsilon$ ).

Разложение $\gamma_{k}$ по $1 / N$ имеет вид

$$
\begin{aligned}
& \gamma_{k}\left(\mathbf{p}_{1}, \mathbf{p}_{2}, \ldots, \mathbf{p}_{k}, y, \epsilon\right)=\gamma_{k}^{(0)}+\frac{1}{N} \gamma_{k}^{(1)}+O\left(\frac{1}{N^{2}}\right), \quad\left(\sum_{l=1}^{k} \mathbf{p}_{l}=0\right), \\
& \gamma_{k}^{(0)}=\exp \left(\frac{a \bar{g}}{16 \pi^{2} \epsilon}\right) L_{k} D^{*} e^{\epsilon d} y^{k(2-\epsilon)} \bar{\phi}_{\mathrm{st}}\left(y\left|\mathbf{p}_{1}\right|\right) \ldots \bar{\phi}_{\mathrm{st}}\left(y\left|\mathbf{p}_{k}\right|\right)+O\left(\epsilon^{2}\right) .
\end{aligned}
$$

Здесь и далее мы обозначаем фурье-образ от $\bar{\phi}_{\mathrm{st}}(\mathbf{x})$ той же буквой $\left(\bar{\phi}_{\mathrm{st}}(\mathbf{p})\right)$, меняя лишь аргумент функции; выражения для констант $L_{k}$ и $D^{*}$ таковы:

$$
\begin{gathered}
L_{k}=\frac{2 \pi^{n / 2}}{\Gamma(n / 2)} \frac{(k-1) ! !}{n(n+2) \ldots(n+k-2)} . \\
D^{*}=2^{(7-4 n) / 2} 3^{5 n / 2} \pi^{-(5+n) / 2} 5^{-5 / 2} \times \\
\times \exp \left(-\frac{4(n+2)}{3}-\frac{7(n+8)}{18}-\frac{R}{12}+\left.U_{\mathrm{reg}}\right|_{\epsilon=0}\right),
\end{gathered}
$$

где

$$
\begin{gathered}
R=-\sum_{l=2}^{\infty} \sum_{p=3}^{\infty} \frac{(l+1)(l+2)(2 l+3)}{p(l+1)^{p}(l+2)^{p}}\left(6^{p}+2^{p}(n-1)\right) \\
U_{\mathrm{reg}}(\epsilon)=\frac{n+8}{6}(\Psi(1)+2-\ln \pi)+\epsilon \frac{n+8}{6}\left(-\ln \pi-\frac{79}{16} \pi-\gamma+\frac{79}{32} \pi \gamma+\frac{79}{32} \pi \ln \pi-\right. \\
\left.-\frac{15}{64} \pi^{3}(\ln \pi+\gamma)+\frac{1}{2} \gamma \ln \pi+\frac{1}{4} \ln ^{2} \pi-\frac{1}{8} \pi^{2}+\frac{1}{4} \gamma^{2}+\frac{15}{32} \pi^{3}+\frac{5}{2}\right)
\end{gathered}
$$

Член $\gamma_{k}^{(0)}$ соответствует гауссовому приближению для интеграла по флуктуациям вокруг инстантона. Член $\gamma_{k}^{(1)}$ содержит первую поправку по $1 / N$ к гауссовому приближению. Как и во многих последующих формулах, мы использовали в (9) экспоненциальную запись первой поправки по $\epsilon$ в выражении для $\gamma_{k}^{(0)}\left(\right.$ см. член $\left.e^{\epsilon d}\right)$. 
В отличие от предыдущих работ [1], [2], [4], [8] в $(4-\epsilon)$-схеме МВ, кроме члена $\gamma_{k}^{(1)}$, в поправочном по $1 / N$ порядке исследуемой асимптотики существенными оказьваются первые поправки по $\epsilon$ ко всем объектам в (7) (к инстантону и $\left.\gamma_{k}^{(0)}\right)$.

Вычисление вычета в $\epsilon=0$ функции $G_{k}^{(N)}$ в асимптотике больших $N$ (в главном и поправочном по $1 / N$ порядках) начнем с формулы (7).

\section{3. ОСОБЕННОСТИ ФОРМАЛИЗМА В $\epsilon$-РАЗЛОЖЕНИИ}

Как и в [3], отличие наших расчетов от расчетов, приведенных в работах [1], [2], [8], [4], будет заключаться в том, что большой параметр $N$ метода стационарной фазы не будет самым большим: мы выделяем определенный с УФ точки зрения объект (полюс по $\epsilon$ ) и только затем используем метод стационарной фазы по $N$. Технически это выражается в выделении из $G_{k}^{(N)}$ УФ-расходимостей (полюсов по $\epsilon$ ), которые будут проявляться в виде расходяшихся при малых $\epsilon$ интегралов по масштабному параметру $y$. При этом поправку к асимптотике больших $N$ дает не только учтенный в [4] член $\gamma_{k}^{(1)}$, но и поправки по $\epsilon$ к инстантону $\left\{\bar{\phi}_{\mathrm{st}}, g_{\mathrm{st}}\right\}$ и к интегралу по гауссовским флуктуациям $\gamma_{k}^{(0)}$. Важным удобством схемы МВ является то, что полюс первого порядка по $\epsilon$ в корреляционных функциях - величина $\left\{G_{k}^{(N)}\right\} / \epsilon$ - содержит всю необходимую информацию о константах ренормировки и критических индексах, поэтому мы можем ограничиться лишь вычислением $\left\{G_{k}^{(N)}\right\}[3],[9]$ (здесь и далее фигурные скобки обозначают вычет в $\epsilon=0)$.

Так как константы ренормировки имеют смысл лишь в рамках теории возмушений, из функционала $J\left(\bar{\phi}_{\mathrm{st}}, \bar{g}_{\mathrm{st}}(\mu y)^{-\epsilon}\right)$, входяшего в $(7)$, выделяется слагаемое

$$
A=\frac{1}{2} \partial \bar{\phi}_{\mathrm{st}} \partial \bar{\phi}_{\mathrm{st}}+\frac{1}{4 !} \bar{g}_{\mathrm{st}} \bar{\phi}_{\mathrm{st}}^{4}+\ln \left(-\bar{g}_{\mathrm{st}}(\mu y)^{-\epsilon}\right),
$$

которое не содержит расходимостей и может быть рассмотрено при анализе выражения (7) как величина в показателе экспоненты: $\exp (-N A)$. Оставшееся слагаемое, контрчленная часть

$$
B=\frac{1}{2}\left(Z_{\phi}^{2}-1\right) \partial \bar{\phi}_{\mathrm{st}} \partial \bar{\phi}_{\mathrm{st}}+\frac{1}{4 !}\left(Z_{\phi}^{4} Z_{g}-1\right) \bar{g}_{\mathrm{st}} \bar{\phi}_{\mathrm{st}}^{4},
$$

содержит константы ренормировки и соответствуюшие им сингулярности по $\epsilon$, которые могут быть рассмотрены только в рамках $\mathrm{TB}$, и при инстантонном анализе (7) должны быть представлены в полиномиальном виде ${ }^{1)}$ :

$$
e^{-N B} \rightarrow \sum_{j} \frac{(-N B)^{j}}{j !}
$$

\footnotetext{
1) В работе [3] было показано, что в УФ-области интегрирования по масштабному параметру вклады левой и правой части данного равенства в ответ, полученный методом стационарной фазы, оказываются различными из-за погрешности вычисления (см. (Т.20)).
} 
При вычислении первой поправки по $1 / N$ используем для $A$ следующие члены разложения в ряд по $\epsilon$ :

$$
A=1+\alpha \epsilon-\epsilon \ln (\mu y)+\frac{\beta \epsilon^{2}}{2 !}+O\left(\epsilon^{3}\right)
$$

где

$$
\begin{gathered}
\alpha=-\frac{\ln \pi}{2}-\frac{\gamma}{2}-1, \\
\beta=-\frac{79}{16} \pi+\frac{56041 \pi^{2}}{3072}-1+\frac{\gamma \ln \pi}{2}+\frac{675 \pi^{6}}{4096}+\frac{15 \pi^{3}}{32}+\frac{15 \pi^{3} \ln \pi}{64}-\frac{79 \pi \gamma}{32}- \\
-\frac{79 \pi \ln \pi}{32}+\frac{15 \pi^{3} \gamma}{64}+\ln \pi+\gamma+\frac{\gamma^{2}}{4}-\frac{3555 \pi^{4}}{1024}+\frac{\ln ^{2} \pi}{4} \simeq-0.05045
\end{gathered}
$$

(по сравнению с [3] мы учли в $A$ слагаемое $\beta \epsilon^{2} / 2$ !). Мы также вычислили инстантон с точностью до членов порядка $\epsilon$ :

$$
\begin{gathered}
\bar{\phi}_{\mathrm{st}}(\mathbf{x})=\frac{\sqrt{3} \exp (-q \epsilon / 2)}{\pi\left(1+\mathbf{x}^{2}\right)}+\epsilon \frac{\sqrt{3}\left(3 \mathbf{x}^{2} \ln \left(\mathbf{x}^{2}\right)+\left(3-\pi^{2}\right) \mathbf{x}^{2}-9+\pi^{2}\right)}{6 \pi\left(1+\mathbf{x}^{2}\right)^{2}}+O\left(\epsilon^{2}\right), \\
\bar{g}_{\mathrm{st}}=-16 \pi^{2} e^{q \epsilon}+O\left(\epsilon^{2}\right), \quad q=\frac{15 \pi^{3}-158 \pi}{64} \simeq-0.4887,
\end{gathered}
$$

а в константах ренормировки ограничились приближением

$$
Z_{g} Z_{\phi}^{4}=1+\frac{a g}{\epsilon 16 \pi^{2}}+\frac{b_{1} g^{2}}{\epsilon\left(16 \pi^{2}\right)^{2}}+\frac{b_{2} g^{2}}{\epsilon^{2}\left(16 \pi^{2}\right)^{2}}, \quad Z_{\phi}^{2}=1+\frac{c g^{2}}{\epsilon\left(16 \pi^{2}\right)^{2}},
$$

где

$$
a=\frac{n+8}{3}, \quad b_{1}=-\frac{5 n+22}{9}, \quad b_{2}=\frac{n^{2}+16 n+64}{9}, \quad c=-\frac{n+2}{36} .
$$

Подставляя в (7) описанные приближенные выражения, получаем

$$
\begin{aligned}
G_{k}^{(N)}= & \frac{(-1)^{N}}{\left(16 \pi^{2}\right)^{N}} N^{N} N^{(k+n+3) / 2} e^{-N-\alpha N \epsilon-\beta N \epsilon^{2} / 2} \times \\
& \times \int_{0}^{\infty} d y y^{k-5} y^{\epsilon(k / 2-1)}(\mu y)^{N \epsilon} \gamma_{k} \sum_{j=0}^{\infty} \frac{1}{j !}\left(-a e^{q \epsilon} \frac{(\mu y)^{-\epsilon}}{\epsilon}\right)^{j}
\end{aligned}
$$

Контрчлен, пропорциональный $a$ из (13), выделен в (14) явно, так как он дает вклад в главный по $1 / N$ порядок (см. (I.19)-(I.21)). Вклады остальных контрчленов из (13) содержатся в $\gamma_{k}^{(1)}$.

Сингулярности по $\epsilon$ в (7), возникаюшие при вычислении $\gamma_{k}$ (например, для слагаемого $\gamma_{k}^{(0)}$ см. член $\exp \left(a \bar{g} /\left(16 \pi^{2} \epsilon\right)\right)$ в формуле $\left.(9)\right)$, также могут быть проанализированы только в рамках ТВ и тоже должны быть представлены в полиномиальном виде в предэкспоненте. Это дает дополнительный вклад к слагаемым суммы по $j$ в (14), так что интеграл в (14) заменится на

$$
\int_{0}^{\infty} d y y^{k-5} y^{\epsilon(k / 2-1)}(\mu y)^{N \epsilon} \bar{\gamma}_{k} \sum_{j=0}^{\infty} \frac{1}{j !}\left(-a e^{q \epsilon} \frac{(\mu y)^{-\epsilon}-1}{\epsilon}\right)^{j},
$$


где $\bar{\gamma}_{k}=\bar{\gamma}_{k}^{(0)}+\bar{\gamma}_{k}^{(1)} / N-$ фллуктуационный интеграл, расходимости которого устранены в соответствии с предписаниями схемы МВ ренормировки. Полученное в результате выражение аналогично (I.21), но содержит необходимые поправки по $1 / N, \epsilon$. Нашей целью является выделение в нем полюса первого порядка по $\epsilon$ и вычисление коэффициента при нем-вычета $\left\{G_{k}^{(N)}\right\}$ - в главном и поправочном по $1 / N$ порядках. Мы проведем эти вычисления для $G_{4}^{(N)}$, т.е. в случае $k=4$. Будет показано, что главный член разложения совпадает с результатами работы [3], а поправочный позволяет уточнить асимптотическое поведение константы ренормировки $Z_{g}$.

\section{4. ОБ ИНТЕГРАЛЕ ПО ФЛУКТУАЦИЯМ \\ В ПРОСТРАНСТВЕ РАЗМЕРНОСТИ $4-\epsilon$}

Вычисление интеграла по флуктуациям $\gamma_{k}$ проводилось в главном порядке разложения по $1 / N$ в работах [1] (при $n=1$ ), [2] (при произвольном $n$ ). Затем в [4] рассматривалась первая поправка по $1 / N$ (в единичной схеме ренормировки). Однако в $(4-\epsilon)$-схеме МВ в ответ для $\left\{G_{4}^{(N)}\right\}$ дает вклад поправка по $\epsilon$ к фолуктуационному интегралу (константа $d$ из (9)). Вычислению этой константы и будут посвящены два следующих раздела.

Итак, обсудим флуктуации вокруг инстантона для выражения

$$
\gamma_{k}=\frac{N^{z}}{\pi i} \oint \frac{d \bar{g}}{\bar{g}} \int d^{n} \vec{u} \frac{\int D \bar{\phi} I y^{2-\epsilon} \bar{\phi}\left(\mathbf{p}_{1} y\right) \ldots y^{2-\epsilon} \bar{\phi}\left(\mathbf{p}_{k} y\right) e^{-N J\left(\bar{\phi}, \bar{g}(\mu y)^{-\epsilon}\right)}}{\operatorname{NORM}}
$$

где

$$
\mathrm{NORM}=\int D \bar{\phi} \exp \left(\frac{-N \partial \bar{\phi} \partial \bar{\phi}}{2}-\frac{N \bar{g} \bar{\phi}^{4}}{4 !}\right) .
$$

Как и в [1], мы включили в $(16)$ множитель $N^{z}($ где $z=(1-n-D) / 2)$, чтобы величина $\gamma_{k}$ не зависела от $N$ при $N \rightarrow \infty$. Отметим здесь, что величина $\gamma_{k}$, выгисленная по формуле (16), в главном порядке разложения по $1 / N$ соответствует слагаемому $\gamma_{k}^{(0)}$ в $(8)$, а первая поправка по $1 / N$ к $\gamma_{k}$ дает, в свою очередь, $\gamma_{k}^{(1)}$.

Замена переменных $\bar{\phi}=\psi / \sqrt{-\bar{g}}$ расцепляет полевые фолуктуации и фолуктуации по параметру $\bar{g}[2]$. Перейдя затем в интегралах (16) и (17) от интегрирования по переменным $\psi, \bar{g}$ к интегрированию по флуктуациям $\delta \psi, \delta \bar{g}$, где

$$
\psi=\bar{\phi}_{\mathrm{st}} \sqrt{-\bar{g}_{\mathrm{st}}}+\delta \psi, \quad \bar{g}=\bar{g}_{\mathrm{st}}+\delta \bar{g},
$$

следует разложить функционал $J$ по этим флуктуациям в окрестности инстантона вплоть до вариаций четвертого порядка [4]. Производя затем обычные для метода перевала растяжения $\delta \psi=\delta \varphi / \sqrt{N}, \delta \bar{g}=\delta g / \sqrt{N}$, получим

$$
\begin{aligned}
\gamma_{k}= & \frac{y^{(2-\epsilon) k}}{\text { NORM }} \frac{N^{z-1 / 2}}{\pi i \bar{g}_{\mathrm{st}}} \int d^{n} \vec{u} \int D \delta \varphi_{\|} \int D \delta \varphi_{\perp} \int_{-\infty}^{+\infty} d \delta g I \bar{\phi}_{\mathrm{st}}\left(p_{1} y\right) \ldots \bar{\phi}_{\mathrm{st}}\left(p_{k} y\right) \times \\
& \times \exp \left(-\frac{(\delta g)^{2}}{2 \bar{g}_{\mathrm{st}}^{2}}-\frac{\delta \varphi_{\|} M_{\|} \delta \varphi_{\|}}{2 !}-\frac{\delta \varphi_{\perp} M_{\perp} \delta \varphi_{\perp}}{2 !}-\frac{J_{3}}{3 ! \sqrt{N}}-\frac{J_{4}}{4 ! N}\right) \times \\
& \times\left[1+\frac{P_{1}}{\sqrt{N}}+\frac{P_{2}}{2 ! N}\right],
\end{aligned}
$$


где символ $\|(\perp)$ означает проектирование (исключение проекции) на направление $\bar{\phi}_{\mathrm{st}}$, операторы $M_{\|}$и $M_{\perp}$ равны, соответственно,

$$
M_{\|}=\frac{1}{\left(-\bar{g}_{\mathrm{st}}\right)}\left(-\Delta^{(D)}+\frac{\bar{g}_{\mathrm{st}} \bar{\phi}_{\mathrm{st}}^{2}}{2 !}\right), \quad M_{\perp}=\frac{1}{\left(-\bar{g}_{\mathrm{st}}\right)}\left(-\Delta^{(D)}+\frac{\bar{g}_{\mathrm{st}} \bar{\phi}_{\mathrm{st}}^{2}}{3 !}\right),
$$

$J_{3}$ и $J_{4}$ - всевозможные вклады вариаций третьего и четвертого порядков функционала $J$ по переменным $\delta \varphi_{\|}, \delta \varphi_{\perp}, \delta g$, зависимость от $N$ при них указана явно. Аналогично, выражение в квадратных скобках в (18) содержит величины $P_{1}$ и $P_{2}$, которыми обозначены вклады от первой и второй вариаций предэкспоненты выражения (16). Отметим, что в (17) достаточно положить

$$
\mathrm{NORM}=\int D \delta \varphi_{\|} D \delta \varphi_{\perp} \exp \left(\frac{\delta \varphi_{\|} \Delta^{(D)} \delta \varphi_{\|}+\delta \varphi_{\perp} \Delta^{(D)} \delta \varphi_{\perp}}{2 \bar{g}_{\mathrm{st}}}\right),
$$

так как все отброшенные в последнем выражении вклады порядка $O(1 / N)$ приводят лишь к диаграммам, содержашим вакуумные петли и обрашаюшимся в нуль в размерной схеме регуляризации в безмассовой модели.

Обозначим через $\left\{\Phi_{\lambda a}^{\|}\right\},\left\{\Phi_{\lambda b}^{\perp}\right\}$ полный набор собственных функций операторов

$$
\bar{g}_{\mathrm{st}}\left(\Delta^{(D)}\right)^{-1} M_{\|}, \quad \bar{g}_{\mathrm{st}}\left(\Delta^{(D)}\right)^{-1} M_{\perp},
$$

соответственно. Индекс $\lambda$ собственной функции фиксирует собственное число (и собственное подпространство) для данной собственной функции, индекс, обозначенный латинской буквой, нумерует функции в данном собственном подпространстве. Разложим флуктуации $\delta \varphi_{\|}, \delta \varphi_{\perp}$ по указанным собственным функциям:

$$
\delta \varphi_{\|}=\sum_{\lambda, a} C_{\lambda a}^{\|} \Phi_{\lambda a}^{\|}, \quad \delta \varphi_{\perp}=\sum_{\lambda, b} C_{\lambda b}^{\perp} \Phi_{\lambda b}^{\perp}
$$

и перейдем от функционального интегрирования по $\delta \varphi_{\|}, \delta \varphi_{\perp}$ к произведению интегралов $\int \prod_{\lambda, a, b} d C_{\lambda a}^{\|} d C_{\lambda b}^{\perp}$ (как в интеграле (18), так и в интеграле (19)).

Среди собственных чисел обсуждаемых операторов некоторые равны нулю при $\epsilon=0$. Интегралы по соответствуюшим коэффициентам разложения - нулевым модам $C_{0 a}^{\|}, C_{0 b}^{\perp}$ $(a=0, \ldots, D, b=1, \ldots,(n-1))$ - снимаются с помощью дельта-функций, содержашихся в $I[1],[2]$. Собственные функции, соответствующие нулевому собственному числу, легко получить, восстановив по выражению (12) (где параметры $y, \mathbf{x}_{0}$ были фиксированы дельта-функциями из $I$ ) при помощи формулы (3) исходное семейство инстантонов, зависящее от произвольных параметров $y, \mathbf{x}_{0}, \vec{u}$. Дифференцируя полученный результат по $y$ или по каждой из компонент векторов $\mathbf{x}_{0}, \vec{u}$ и снова полагая $y=1, \mathbf{x}_{0}=0$, получим

$$
\Phi_{00}^{\|}=\vec{u} \frac{\partial \bar{\phi}_{\mathrm{st}}}{\partial y}, \quad \Phi_{0 a^{\prime}}^{\|}=\vec{u} \frac{\partial \bar{\phi}_{\mathrm{st}}}{\partial \mathbf{x}_{0}^{a^{\prime}}}, \quad \Phi_{0 b}^{\perp}=\vec{e}_{b}\left|\bar{\phi}_{\mathrm{st}}\right|,
$$

где $a^{\prime}=1, \ldots, D, b=1, \ldots, n-1$ и $\vec{e}_{b}$ - ортонормированный базис в изотопическом подпространстве, ортогональном направлению инстантона $\vec{u}$. 


\section{5. ВЫЧИСЛЕНИЕ ПОПРАВКИ ПО $\epsilon$ К ФЛУКТУАЦИОННОМУ ИНТЕГРАЛУ В ГАУССОВСКОМ ПРИБЛИЖЕНИИ}

Пользуясь введенными в предыдушей главе обозначениями, вычислим теперь константу $d$ в (9). При этом нам достаточно ограничиться гауссовым приближением для функционального интеграла, т. е. рассматривать выражение (18) в главном порядке по $1 / N$.

Проиллюстрируем, как "работают" в старшем по $1 / N$ порядке дельта-функции в формуле для $I$ на примере $\delta^{D}\left(-\bar{g} \int d^{D} \mathbf{x x}_{\mu} \bar{\phi}^{4}(\mathbf{x}) / 4 !\right)$. Раскладывая аргумент дельтафункции в ряд по $1 / N$ и переходя к переменным $C_{\lambda}^{\|}, C_{\lambda}^{\perp}$, получим для вклада обсуждаемой дельта-функции в (16) (в главном по $1 / N$ порядке) выражения вида

$$
N^{\frac{D}{2}} \prod_{\mu=1}^{D} \delta\left(\sum_{a^{\prime}=1}^{D} C_{0 a^{\prime}}^{\|} B_{\mu a^{\prime}}^{0}+\sum_{\lambda \neq 0} \sum_{c} C_{\lambda c}^{\|} B_{\mu c}^{\lambda}\right)
$$

где

$$
B_{\mu c}^{\lambda}=\int d^{D} \mathbf{x} \frac{\sqrt{-\bar{g}_{\mathrm{st}}} \bar{\phi}_{\mathrm{st}}^{3} \Phi_{\lambda c}^{\|} \mathbf{x}^{\mu}}{3 !} ; \quad B_{\mu a^{\prime}}^{0}=\frac{1}{\sqrt{-\bar{g}_{\mathrm{st}}}} \delta_{\mu a^{\prime}}
$$

Теперь видно, что коэффициенты $C_{0 a^{\prime}}^{\|}$, которые входят в числитель выражения (16), фиксируются обсуждаемой дельта-функцией, а соответствуюшие им интегралы в числителе формулы (16) снимаются.

Рассмотренная в качестве примера дельта-функция приводит в главном по $1 / N$ порядке к множителю $\operatorname{det}\left(B_{\mu a^{\prime}}^{0}\right)^{-1}=\left(-\bar{g}_{\mathrm{st}}\right)^{D / 2}$. Интеграл по $C_{0}^{\|}$в нормирующем знаменателе (19) является гауссовым и равен

$$
\prod_{a=1}^{D} \sqrt{\frac{2 \pi\left(-\bar{g}_{\mathrm{st}}\right)}{\sum_{\nu=1}^{D}\left(\partial_{\nu} \Phi_{0 a}^{\|}, \partial_{\nu} \Phi_{0 a}^{\|}\right)}}
$$

где символ скалярного произведения $(\cdot, \cdot)$ здесь и далее означает интегрирование по $D$-мерной координате.

Рассматривая аналогично остальные члены $I$ и выполняя гауссово интегрирование по $\delta g, C_{\lambda a}^{\|}, C_{\lambda a}^{\perp}$, а также интегрирование по $\vec{u}$, получим для $\bar{\gamma}_{4}$ в главном по $1 / N$ порядке

$$
\bar{\gamma}_{k}^{0}=\frac{e^{U_{\operatorname{reg}} L_{k}}}{\sqrt{2 \pi}}\left(\Pi(1) \Pi(3)^{n-1}\right)^{-1 / 2} \sqrt{\sum_{b=1}^{n-1} \frac{\left(\partial_{\nu} \Phi_{0 b}^{\perp}, \partial_{\nu} \Phi_{0 b}^{\perp}\right)}{2 \pi}} \prod_{a=0}^{D} \sqrt{\sum_{\nu=1}^{D} \frac{\left(\partial_{\nu} \Phi_{0 a}^{\|}, \partial_{\nu} \Phi_{0 a}^{\|}\right)}{2 \pi}},
$$

где

$$
\Pi(\alpha)=\prod_{\substack{S \\ \alpha \mu_{s} \neq 1}}\left|1-\frac{1}{\alpha \mu_{s}}\right| \prod_{s^{\prime}} \exp \left[\frac{1}{\alpha \mu_{s^{\prime}}}+\frac{1}{2 \alpha^{2} \mu_{s^{\prime}}^{2}}\right]
$$

Здесь мы ввели $\mu_{s}-$ собственные числа задачи

$$
\Delta^{(D)} \Psi_{s}(\mathbf{x})=\mu_{s}\left(\frac{\bar{g}_{\mathrm{st}} \bar{\phi}_{\mathrm{st}}^{2}}{2 !}+12 \bar{\rho} \bar{\phi}_{\mathrm{st}}^{2} \ln \left(\mathbf{x}^{2}\right)\right) \Psi_{s}(\mathbf{x}), \quad \bar{\rho}=-\frac{\bar{g}_{\mathrm{st}} \epsilon}{48} .
$$


Произведения функций П(1), П(3) в (22) возникают при гауссовом интегрировании по всем модам, кроме нулевых. Как и в работах [1], [2], [4], [8], расходимости однопетлевых диаграмм целиком вычтены множителями $\exp \left(\left(\alpha \mu_{s}\right)^{-1}+\left(\alpha \mu_{s}\right)^{-2} / 2\right)$. Такое вычитание соответствует "единичной” схеме ренормировки. Для того чтобы результат был справедлив в схеме MB, необходимо домножить его на $e^{U_{\text {reg }}}$, где $U_{\text {reg }}$ представляет собой конечную часть упомянутых диаграмм. В работе [3] подробно описан способ вычисления константы $U_{\mathrm{reg}}$. Этот результат приведен выше (см. формулу (10)) с необходимой по сравнению с [3] поправкой по $\epsilon$. Как уже отмечалось, вклады, соответствуюшие нулевым модам (т. е. таким, что $\left.\alpha \mu_{s}=1+O(\epsilon)\right)$ должны быть исключены из произведения по $s$. Кроме того, оказывается, что одно из собственных чисел $\left(\mu_{s}=1 / 3+O(\epsilon)\right)$ нарушает положительную определенность матрицы вторых производных функционала $J$. Для интегрирования по соответствующей моде произведен разворот контура в комплексной плоскости, уничтожающий мнимую единицу в (18) и приводящий к появлению модуля в выражении для $\Pi(\alpha)$.

Зависимость от $\epsilon$ в выражении (22) присутствует в скалярном произведении и нулевых модах $\Phi_{0}^{\|}, \Phi_{0}^{\perp}$. Зависимость эту легко восстановить явно при помощи формул (20):

$$
\begin{aligned}
& \sum_{\nu=1}^{D}\left(\partial_{\nu} \Phi_{00}^{\|}, \partial_{\nu} \Phi_{00}^{\|}\right)=\frac{12}{5}+\epsilon\left(-\frac{6}{5} \ln \pi-\frac{24}{5}-\frac{12}{5} q+\frac{6}{5} \Psi(1)\right)+O\left(\epsilon^{2}\right), \\
& \sum_{\nu=1}^{D}\left(\partial_{\nu} \Phi_{0 a}^{\|}, \partial_{\nu} \Phi_{0 a}^{\|}\right)=\frac{12}{5}+\epsilon\left(-\frac{6}{5} \ln \pi-\frac{12}{5} q-8+\frac{4 \pi^{2}}{5}+\frac{6}{5} \Psi(1)\right)+O\left(\epsilon^{2}\right), \\
& \sum_{\nu=1}^{n-1}\left(\partial_{\nu} \Phi_{0 b}^{\perp}, \partial_{\nu} \Phi_{0 b}^{\perp}\right)=4 .
\end{aligned}
$$

Однако в (22) также имеется зависимость от $\epsilon$, содержашаяся в собственных числах $\mu_{s}$ задачи (24). Собственные числа невозмушенной задачи $(\epsilon=0)$ хорошо известны [2]:

$$
\begin{gathered}
\mu_{s}^{(0)}=\frac{(l+n)(l+n+1)}{6}, \quad s \equiv[l, n], \quad l=0,1,2, \ldots, \quad n=1,2,3, \ldots, \\
V=\frac{\Gamma(l+D-2)(D+2 l-2)}{\Gamma(D-1) \Gamma(l+1)} \equiv(l+1)^{2}+\epsilon \aleph(l)+O\left(\epsilon^{2}\right),
\end{gathered}
$$

где $V$ - вырождение собственных чисел $\mu_{s}$ и

$$
\aleph=\frac{\Psi(3)(l+1)^{2}}{2}-\frac{l+1}{1}-(l+1)^{2} \Psi(l+2) .
$$

Следуя методу, описанному в [8], мы вычислили также собственные функции данной (невозмущенной) задачи:

$$
\Psi_{l, n}^{0}(\mathbf{x})=Y_{l}(\mathbf{x})\left(\frac{|\mathbf{x}|}{\mathbf{x}^{2}+1}\right)^{n+l} \sum_{i=1}^{n / 2} A_{n, i}\left(|\mathbf{x}|^{-n+2 i-2}-|\mathbf{x}|^{n-2 i}\right)
$$


при четном $n$,

$$
\Psi_{l, n}^{0}(\mathbf{x})=Y_{l}(\mathbf{x})\left(\frac{|\mathbf{x}|}{\mathbf{x}^{2}+1}\right)^{n+l}\left(\sum_{i=1}^{(n-1) / 2} A_{n, i}\left(|\mathbf{x}|^{-n+2 i-2}-|\mathbf{x}|^{n-2 i}\right)+\frac{A_{n,(n+1) / 2}}{|\mathbf{x}|}\right)
$$

при нечетном $n$. Здесь $Y_{l}(\mathbf{x})$ - сферические функции, коэффициенты $A_{n, i}$ задаются формулой

$$
A_{n, i}=\frac{(-1)^{i+1}(n-1) !}{(n-i) !(i-1) !} \frac{(l+n) !(l+1) !}{(l+n-i+1) !(l+i) !} .
$$

Будем искать собственные числа в виде $\mu_{s}=\mu_{s}^{0}+\epsilon \bar{\mu}_{s}+O\left(\epsilon^{2}\right)$, интересуясь первой поправкой по $\epsilon$. Чтобы найти $\bar{\mu}_{s}$, домножим $(24)$ на $\Psi_{s}$ и проинтегрируем по $D$-мерной координате. Подставим затем в (24) разложение в ряд для инстантона (12). Используем также явное выражение для оператора Лапласа в сферических координатах (см., например, [2])

$$
\Delta^{(D)}=\frac{d^{2}}{d r^{2}}+\frac{D-1}{r} \frac{d}{d r}-\frac{L^{2}}{r^{2}}
$$

где $L^{2}$ - оператор Казимира $D$-мерной врашательной группы, который имеет собственные числа $l(l+D-2), l=0,1, \ldots$ с вырождением $V(26)$. Представим $D$-мерньй оператор Лапласа, действуюший на собственную функцию (с собственным числом $l$ ) упомянутого оператора Казимира, в виде

$$
\Delta^{(D)}=\Delta^{(4)}+\epsilon \bar{\Delta}, \quad \Delta^{(4)}=\frac{d^{2}}{d r^{2}}+\frac{3}{r} \frac{d}{d r}-\frac{l(l+2)}{r^{2}}, \quad \bar{\Delta}=-\frac{1}{r} \frac{d}{d r}+\frac{l}{r^{2}} .
$$

Кроме того, напишем формальное разложение для собственных функций $\Psi_{s}=\Psi_{s}^{0}+\epsilon \bar{\Psi}_{s}$. Слагаемые в последнем равенстве следует понимать в смысле обобщенных функций, имея в виду естественное продолжение функции $\Psi_{s}^{0}$ в пространство размерности $4-\epsilon$ (см., например, [12]). Выделив теперь линейные по $\epsilon$ члены и учитывая равенство

$$
\mu_{s} \int d^{4} \mathbf{x} \Psi_{s}^{0} \frac{\bar{g}_{\mathrm{st}} \bar{\phi}_{\mathrm{st}}^{2}}{2 !} \bar{\Psi}_{s}=\int d^{4} \mathbf{x} \Psi_{s}^{0} \Delta^{(4)} \bar{\Psi}_{s}
$$

(которое легко может быть доказано, если разложить функцию $\bar{\Psi}_{s}$ по полному набору собственных функций $\left\{\Psi_{s}^{0}\right\}$ и учесть, что последние взаимно ортогональны относительно скалярного произведения $\left.\sum_{\alpha=1}^{4} \int d^{4} \mathbf{x} \partial_{\alpha} \Psi_{s}^{0} \partial_{\alpha} \Psi_{s^{\prime}}^{0}\right)$, получим, наконец, первую поправку по $\epsilon$ для собственных чисел задачи (24):

$$
\bar{\mu}_{s}=\mu_{s}^{0} \frac{\left(\Psi_{s}^{0}, \bar{\Delta} \Psi_{s}^{0}\right)}{\left(\Psi_{s}^{0}, \Delta^{(4)} \Psi_{s}^{0}\right)}-\left(\mu_{s}^{0}\right)^{2} \frac{\left(\chi \Psi_{s}^{0}, \Psi_{s}^{0}\right)}{\left(\Psi_{s}^{0}, \Delta^{(4)} \Psi_{s}^{0}\right)} .
$$

Здесь $\chi$ определяется равенством

$$
12 \bar{\rho} \bar{\phi}_{\mathrm{st}}^{2} \ln \mathbf{x}^{2}+\frac{\bar{g}_{\mathrm{st}} \bar{\phi}_{\mathrm{st}}^{2}}{2 !}=\left.\frac{\bar{g}_{\mathrm{st}} \bar{\phi}_{\mathrm{st}}^{2}}{2 !}\right|_{\epsilon=0}+\epsilon \chi+O\left(\epsilon^{2}\right)
$$


тем самым,

$$
\chi=-8 \frac{3 \ln \left(\mathbf{x}^{2}\right) \mathbf{x}^{2}+3 \mathbf{x}^{2}-9+\pi^{2}-\pi^{2} \mathbf{x}^{2}}{\left(\mathbf{x}^{2}+1\right)^{3}}+\frac{12 \ln \mathbf{x}^{2}}{\left(\mathbf{x}^{2}+1\right)^{2}} .
$$

Теперь несложно учесть зависимость от $\epsilon$ произведений по $s$ и $s^{\prime}$ в $(22)$ :

$$
\begin{aligned}
\ln (\Pi(\alpha))= & \left.\ln (\Pi(\alpha))\right|_{\epsilon=0}+\epsilon\left(\sum _ { \alpha \mu _ { l , n } ^ { 0 } \neq 1 } \left[(l+1)^{2} \frac{\bar{\mu}_{l, n}}{\alpha\left(\mu_{l, n}^{0}\right)^{2}-\mu_{l, n}^{0}}+\right.\right. \\
& \left.+\ln \left|1-\frac{1}{\alpha \mu_{l, n}^{0}}\right| \aleph(l)\right]-\sum_{l, n}\left[(l+1)^{2}\left(\frac{\bar{\mu}_{l, n}}{\alpha\left(\mu_{l, n}^{0}\right)^{2}}+\frac{\bar{\mu}_{l, n}}{\alpha^{2}\left(\mu_{l, n}^{0}\right)^{3}}\right)-\right. \\
& \left.\left.-\left(\frac{1}{\alpha \mu_{l, n}^{0}}+\frac{1}{2 \alpha^{2}\left(\mu_{l, n}^{0}\right)^{2}}\right) \aleph(l)\right]\right) .
\end{aligned}
$$

С помощью формулы (27) мы получили численные значения поправок $\bar{\mu}_{s}$. Их подстановка в (28) дает

$$
\begin{aligned}
& \left.\ln \Pi(1) \simeq \ln \Pi(1)\right|_{\epsilon=0}-4.90 \epsilon+O\left(\epsilon^{2}\right), \\
& \left.\ln \Pi(3) \simeq \ln \Pi(3)\right|_{\epsilon=0}-0.340 \epsilon+O\left(\epsilon^{2}\right) .
\end{aligned}
$$

Окончательно, собрав в константу $d$ все поправки по $\epsilon$ из (22), с учетом выражения для $U_{\text {reg }}($ см. формулу (10)), имеем

$$
\begin{aligned}
d= & \frac{4.90}{2}+\frac{(n-1) 0.340}{2}-\frac{3}{2} \ln \pi+\frac{395}{32} \pi-\frac{75}{64} \pi^{3}-\frac{46}{3}+\frac{4}{3} \pi^{2}-\frac{5}{2} \gamma+\ln \frac{5}{6}+ \\
& +\frac{n+8}{6}\left(-\ln \pi-\frac{79}{16} \pi-\gamma+\frac{79}{32} \pi \gamma+\frac{79}{32} \pi \ln \pi-\frac{15}{64} \pi^{3} \gamma-\right. \\
& \left.-\frac{15}{64} \pi^{3} \ln \pi+\frac{1}{2} \gamma \ln \pi+\frac{1}{4} \ln ^{2} \pi-\frac{1}{8} \pi^{2}+\frac{1}{4} \gamma^{2}+\frac{15}{32} \pi^{3}+\frac{5}{2}\right) \simeq-0.59+0.195 n
\end{aligned}
$$

\section{6. АСИМПтОТИКА $G_{4}^{(N)}$ ПРИ БОЛЬШИХ $N$}

6.1. Вклад гауссовой части интеграла по флуктуациям. В данном разделе мы ограничимся рассмотрением вклада в исследуемую асимптотику, связанного с гауссовым приближением для интеграла по флуктуациям, т.е. положим пока в (7) $\gamma_{4} \equiv \gamma_{4}^{(0)}$. Этот вклад является наиболее интересным, так как демонстрирует взаимодействие параметров $y, N$ и $\epsilon$ в схеме МВ, и его рассмотрение отсутствует в предыдуших работах (посвященных другим схемам). Для дальнейших вычислений нам понадобится входящее в $\gamma_{4}^{(0)}$ фурье-преобразование в пространстве размерности $D$ функции $\bar{\phi}_{\mathrm{st}}(x)(12)$. Приведем результат в пределе $\epsilon, p \rightarrow 0$ :

$$
\begin{gathered}
\bar{\phi}_{\mathrm{st}}(\mathbf{p})=\frac{4 \sqrt{3} \pi}{\mathbf{p}^{2}}\left(e^{Q \epsilon}|\mathbf{p}|^{\epsilon}+\epsilon\left[\Psi^{\prime}(1)-\frac{\ln \left(\mathbf{p}^{2}\right)}{2}+\frac{3-\pi^{2}}{6}\right]+O\left(\epsilon^{2}\right)\right)+O\left(\ln \left(\mathbf{p}^{2}\right)\right), \\
Q=-\Psi(1)-\frac{3 \ln 2}{2}-\frac{\ln \pi}{2}-\frac{q}{2} \simeq-0.791,
\end{gathered}
$$


здесь и далее $\Psi(x)$ - логарифмическая производная гамма-функции.

Разложим выражение $\left((\mu y)^{-\epsilon}-1\right)^{j}$ в (15) по формуле бинома Ньютона, введя дополнительную сумму по $m$ от нуля до $j$. Все образовавшиеся интегралы берутся интегрированием по частям:

$$
\begin{aligned}
& \int \frac{d y}{y} y^{\epsilon} y^{(N-u) \epsilon}\left[y^{2-\epsilon} \bar{\phi}_{\text {st }}\left(y\left|\mathbf{p}_{1}\right|\right) \ldots y^{2-\epsilon} \bar{\phi}_{\text {st }}\left(y\left|\mathbf{p}_{4}\right|\right)\right] \simeq \\
& \simeq \frac{(4 \sqrt{3} \pi)^{4}}{\mathbf{p}_{1}^{2} \mathbf{p}_{2}^{2} \mathbf{p}_{3}^{2} \mathbf{p}_{4}^{2}}\left(\frac{1}{(N-u) \epsilon}+\frac{f}{(N-u)}+\frac{3}{(N-u)^{2} \epsilon}\right) \\
& f=2-6 \ln 2-2 \ln \pi+\frac{79 \pi}{16}-\frac{2 \pi^{2}}{3}-\frac{15 \pi^{3}}{32} \simeq-10.05
\end{aligned}
$$

По сравнению с выражениями (I.22), (І.23) мы учли в (30) дополнительные поправки по $1 / N$ и $\epsilon$. Последующие поправки к (30) дают в исследуемую асимптотику вкладпорядка $O\left(1 / N^{2}\right)$, т.е. являются несушественными. Затем с помошью формулы

$$
\sum_{m=0}^{j} \frac{(-1)^{m} C_{j}^{m}}{(N-m)^{z}}=(-1)^{j}\left(\frac{(j+z-1) !}{(z-1) ! N^{j+z}}+\frac{2(j+z) !}{(z-1) ! N^{j+z+1}}\right)+O\left(\frac{1}{N^{j+z+2}}\right)
$$

при $z=1,2$ нетрудно осушествить суммирование по $m$. Получившаяся формула отражает явно зависимость $G_{4}^{(N)}$ в $(14)$ от параметра $\epsilon$ : в каждом слагаемом суммы по $j$ полюсы различного порядка по $\epsilon$ умножены на экспоненциальный фактор $\exp (\alpha N \epsilon+$ $\left.\beta N \epsilon^{2} / 2+d \epsilon+q j \epsilon\right)$. Теперь необходимо в каждом слагаемом суммы по $j$ выделить полюс первого порядка по $\epsilon$ и просуммировать все вклады. Окончательно получим

$$
\frac{\left\{G_{4}^{(N)}\right\}_{0}}{\epsilon} \approx \frac{C_{4}^{(N)}}{\left(16 \pi^{2}\right)^{N}} \frac{e^{\alpha a}}{|\mathbf{p}|^{8}} \frac{1}{N \epsilon}\left(1-\frac{W_{4}}{N}\right)
$$

где

$$
\begin{gathered}
W_{4}=d a+q a+q a^{2} \alpha+\frac{\beta a^{2}}{2}-5-5 \alpha a+f a \simeq-3.58+1.48 n+0.16 n^{2}, \\
C_{4}^{(N)}=(-1)^{N} D^{*} L_{4}(4 \sqrt{3} \pi)^{4} N^{N} N^{(n+7) / 2} e^{-N},
\end{gathered}
$$

символом $\left\{G_{4}^{(N)}\right\}_{0}$ обозначен вклад слагаемого $\gamma_{4}^{(0)}$ в вычет в $\epsilon=0$ частично ренормированной функции Грина $G_{4}^{(N)}$, который и обсуждался в данном пункте.

6.2. Асимптотика $G_{4}^{(N)}$ при больших $N$ : поправка к гауссовому приближению в интеграле по флуктуациям. В данном разделе мы обсудим вклад в исследуемую асимптотику, связанный с учетом первого поправочного члена метода стационарной фазы к интегралу по флуктуациям, т.е. положим теперь в $(7) \gamma_{4} \equiv \gamma_{4}^{(1)} / N$. Такой вклад содержит дополнительную малость порядка $1 / N$ по сравнению со вкладом, рассмотренным в предыдушем пункте, что дает нам возможность не учитывать здесь поправки порядка $\epsilon$ к конечным при $\epsilon \rightarrow 0$ объектам. Первый поправочный член к корреляционной функции $G_{4}^{(N)}$ вычислялся в работе [4] при использовании размерной 
регуляризации и единичной схемы ренормировки. Для поправки по $1 / N$ к асимптотике функции Грина $G_{4}^{(N)}$ (и, соответственно, к вершинной функции $\Gamma_{4}^{(N)}$ ) в [4] должен был быть получен ответ вида $\tilde{A} \ln ^{2}\left(\mathbf{p}^{2} / \mu^{2}\right)+\widetilde{B} \ln \left(\mathbf{p}^{2} / \mu^{2}\right)+\widetilde{C}$. К сожалению, в [4] ответ для асимптотики коэффициента $G_{4}^{(N)}$ не приведен. Приводится лишь асимптотика функции Гелл-Манна-Лоу $\beta(g)$, которая связана с вершинной функцией $\Gamma_{4}$ соотношением [1]

$$
\beta(g)=-\frac{\partial \Gamma_{4}}{\partial \ln \left(\mathbf{p}^{2} / \mu^{2}\right)},
$$

где $\mu$ - симметричная импульсная точка вычитания в единичной схеме ренормировки. При вычислении $\beta^{(N)}$ на основании $\Gamma_{4}^{(N)}$ член из $\Gamma_{4}^{(N)}$, пропорциональньй $\ln ^{2}\left(\mathbf{p}^{2} / \mu^{2}\right)$, который, как известно, не дает вклада в $\beta$, был отброшен. Затем в работе [4] была проведена замена переменных

$$
\frac{\partial}{\partial \ln \left(p^{2} / \mu^{2}\right)} \rightarrow-\frac{\partial}{\partial \ln (\mu y)^{2}}
$$

после чего осушествлялось интегрирование по частям под знаком интеграла по $y$.

Чтобы восстановить результат работы [4] для функции Грина $G_{4}^{(N)}$, мы рассмотрели вклады контрчленов

$$
\left(a \frac{4 \bar{g}_{\mathrm{st}}^{2} \bar{\phi}_{\mathrm{st}}^{3} \delta \bar{\phi}}{N \epsilon 16 \pi^{2} 4 !}\right)^{2}, \quad b_{2} \frac{\bar{g}_{\mathrm{st}}^{3} \bar{\phi}_{\mathrm{st}}^{4}}{N \epsilon^{2}\left(16 \pi^{2}\right)^{2} 4 !},
$$

которые и соответствуют отброшенному коэффициенту $\tilde{A}$. Коэффициент $\widetilde{B}$ при $\ln \left(p^{2} / \mu^{2}\right)$ состоит из вклада контрчленов

$$
b_{1} \frac{\bar{g}_{\mathrm{st}}^{3} \bar{\phi}_{\mathrm{st}}^{4}}{N \epsilon\left(16 \pi^{2}\right)^{2} 4 !}, \quad c \frac{\bar{g}_{\mathrm{st}}^{3} \bar{\phi}_{\mathrm{st}}^{4}}{N \epsilon\left(16 \pi^{2}\right)^{2} 4 !}
$$

и вклада $X$ диаграмм, связанных с вариациями по флуктуациям функции $I$. С помощью приведенного в [4] результата для функции $\beta$ мы восстановили коэффициент $X$ :

$$
-a\left(X+2\left(b_{1}-2 c\right)\right)=-\frac{n^{2}+10 n+36}{6}
$$

(ср. правую часть последнего равенства с выражением для $I_{1}$ в [4]). Последний шаг конечная ренормировка результатов работы [4], чтобы преобразовать их к схеме МВ был осушествлен нами следующим образом. В единичной схеме параметры $a, b_{1}, c$ в $(13)$ оказываются функциями $\epsilon$, что добавляет некоторые конечные вклады к константам ренормировки (13) в пределе малых $\epsilon$. Чтобы ликвидировать эти вклады мы, воспользовавшись приведенными в [4] выражениями для констант ренормировки в единичной схеме, положили, что симметричная точка вычитаний есть $\mu=2(\pi \exp (\Psi(1)+2))^{1 / 2}$. Это привело к исчезновению линейного по $\epsilon$ члена в функции $a(\epsilon)$ и, тем самым, уничтожило соответствующий вклад в константы ренормировки (13) в пределе малых $\epsilon$. Кроме того, мы домножили результат, приведенный в работе [4], на $\exp \left(U^{(1)} / N\right)=1+U^{(1)} / N+$ $O\left(1 / N^{2}\right)$

$$
U^{(1)}=\left(-\frac{1}{9}+\frac{1}{216} \pi^{2}\right) n^{2}+\left(\frac{2}{27} \pi^{2}+\frac{5}{9} \tau-\frac{35}{36}-\frac{1}{36} \gamma\right) n+\frac{22}{9} \tau+\frac{8}{27} \pi^{2}-\frac{1}{18} \gamma-\frac{7}{2},
$$

3 Теоретическая и математическая физика, т. 143, № 2, 2005 г. 
здесь и далее $\tau \simeq 0.749$ [4]. Это уничтожило вклад линейных по $\epsilon$ членов зависимостей $b_{1}(\epsilon), c(\epsilon)$ в константы ренормировки (13). Окончательно преобразованный к схеме МВ результат работы [4] для вклада в функцию Грина, связанного с членом $\gamma^{(1)}$, имеет следуюший вид:

$$
\begin{aligned}
G_{4}^{(N)}( & \left.\gamma_{4} \rightarrow \frac{\gamma_{4}^{(1)}}{N}\right)=\frac{C_{4}^{(N)}}{\left(16 \pi^{2}\right)^{N}} \frac{e^{-\alpha N \epsilon}}{(4 \sqrt{3} \pi)^{4}} \int_{0}^{\infty} \frac{d y}{y}(\mu y)^{N \epsilon}\left(-\frac{\left[y^{2} \bar{\phi}_{\mathrm{st}}(y|\mathbf{p}|)\right]^{4}}{N} \times\right. \\
& \times\left[\frac{a^{2}\left((\mu y)^{-\epsilon}-1\right)^{2}}{\epsilon^{2}}+\frac{b_{2}\left((\mu y)^{-2 \epsilon}-1\right)^{2}}{\epsilon^{2}}+\frac{X\left((\mu y)^{-\epsilon}-1\right)}{\epsilon}+\right. \\
& \left.+\frac{\left(b_{1}-2 c\right)\left((\mu y)^{-2 \epsilon}-1\right)}{\epsilon}+\widetilde{C}-U^{(1)}\right]+\frac{\left[y^{2} \bar{\phi}_{\mathrm{st}}(y|\mathbf{p}|)\right]^{3}}{N \mathbf{p}^{2}}(S-2 \ln y)+ \\
& \left.+\frac{24}{5} \frac{\left[y^{2} \bar{\phi}_{\mathrm{st}}(y|\mathbf{p}|)\right]^{2}}{N \mathbf{p}^{4}}\right) \sum_{j=0}^{\infty} \frac{(-a)^{j}}{j !} \frac{\left((\mu y)^{-\epsilon}-1\right)^{j}}{\epsilon^{j}}
\end{aligned}
$$

где

$$
\begin{gathered}
S=-\frac{1}{80} \frac{1289+55 n}{n+8}+4 \ln 2-3 \gamma-\frac{T}{16} \\
T \equiv \int_{0}^{\infty} d t_{1} \int_{0}^{\infty} d t_{2} \int_{0}^{\infty} d t_{3} \ln t_{3} \frac{1}{\left(t_{1} t_{2}+t_{2} t_{3}+t_{1} t_{3}\right)^{2}} \times \\
\quad \quad \times \exp \left(-\frac{7 t_{1} t_{3} / 3+4 t_{3}^{2} / 3+t_{1}^{2}+t_{1} t_{2}+t_{2} t_{3}}{4\left(t_{1} t_{2}+t_{2} t_{3}+t_{1} t_{3}\right) /\left(t_{1}+t_{3}\right)}\right) \approx 17.6 \\
\widetilde{C}=-\frac{(180 \gamma-219-90 \ln 3) n^{3}}{360(n+8)^{2}}+ \\
-\frac{\left(-1560 \ln 3+12+35 \pi^{2}-120 \ln 2-600 \tau+3240 \gamma\right) n^{2}}{360(n+8)^{2}}+ \\
+\frac{\left(22272+20880 \gamma+560 \pi^{2}-9840 \ln 3-1200 \ln 2-7440 \tau\right) n}{360(n+8)^{2}}+ \\
+\frac{86880+2240 \pi^{2}+51840 \gamma-1920 \ln 2-21120 \tau-24960 \ln 3}{360(n+8)^{2}}
\end{gathered}
$$

а $X$ задается соотношением (33).

Аналогично разделу 6.1 найдем вклад в $G_{4}^{(N)}$ выражения (34). Вместо (30), (32) нам потребуется использовать следующие формулы (справедливые в требуемом порядке разложения по $1 / N$ и $\epsilon)$ :

$$
\begin{aligned}
\int \frac{d y}{y} y^{(N-u) \epsilon}\left[y^{2} \bar{\phi}_{\mathrm{st}}(y|\mathbf{p}|)\right]^{z} & \simeq \frac{(4 \sqrt{3} \pi)^{z}}{|\mathbf{p}|^{2 z}} \frac{1}{(N-u) \epsilon}, \quad z=2,3,4, \\
\int \frac{d y}{y} y^{(N-u) \epsilon} \ln y\left[y^{2} \bar{\phi}_{\mathrm{st}}(y|\mathbf{p}|)\right]^{3} & \simeq-\frac{(4 \sqrt{3} \pi)^{3}}{|\mathbf{p}|^{6}} \frac{1}{(N-u)^{2} \epsilon^{2}} .
\end{aligned}
$$


Окончательно для вклада $\gamma^{(1)}$ в полюс первого порядка по $\epsilon$ функции Грина $G_{4}^{(N)}$ получим

$$
\frac{\left\{G_{4}^{(N)}\right\}_{1}}{\epsilon} \approx \frac{C_{4}^{(N)}}{\left(16 \pi^{2}\right)^{N}} \frac{e^{\alpha a}}{|\mathbf{p}|^{8} N^{2} \epsilon} \widetilde{W}_{4}
$$

где

$$
\begin{aligned}
\widetilde{W}_{4} & =\alpha^{2} a^{2}+4 b_{2} \alpha^{2}-X \alpha-2 \alpha\left(b_{1}-2 c\right)+\widetilde{C}-U^{(1)}+\frac{\sqrt{3}(S-2 \alpha)}{12 \pi}+\frac{1}{10 \pi^{2}} \simeq \\
& \simeq \frac{7879.39+3982.50 n+755.17 n^{2}+63.07 n^{3}+1.924 n^{4}}{(n+8)^{2}} .
\end{aligned}
$$

Суммируя $(32)$ и (35), находим вычет в $\epsilon=0$ частично ренормированной функции $G_{4}^{(N)}$ в $(4-\epsilon)$-схеме МВ в главном и поправочном члене $1 / N$-разложения.

\section{7. КОНСТАНТЫ РЕНОРМИРОВКИ И КРИТИЧЕСКИЕ ИНДЕКСЫ}

Конечность ренормированной функции $G_{4 R}$ позволяет вычислить асимптотику константы ренормировки $Z_{g}$.

Для ренормированной вершинной функции Грина вплоть до $N$-го порядка по $g$ имеем

$$
G_{4 R}=Z_{\varphi}^{4}\left[-\searrow\left\langle g Z_{g}+\frac{(n+8)}{6} \circlearrowright g^{2} Z_{g}^{2}+\cdots+g^{N} Z_{g}^{N} G_{4}^{(N)}\right]\right.
$$

В последнем равенстве подразумевается, что диаграммы содержат лишь поверхностные расходимости (при их вычислении учитываются контрчлены вплоть до порядка $g^{N-1}$ ), а первый полюс констант ренормировки $Z_{g}, Z_{\varphi}$ содержит лиш слагаемые, соответствуюшие контрчленам порядка $g^{N}$.

Вклады в правую часть (36) порядка $g^{N}$, имеющие полюсы первого порядка по $\epsilon$, должны сокрашаться. Это приводит к следуюшему выражению для константы ренормировки $Z_{g}$ в асимптотике больших $N$ (см. (I.30)-(I.31)):

$$
-\frac{\left\{Z_{g}^{(N-1)}\right\}}{\epsilon}+\frac{\left\{G_{4}^{(N)}\right\}}{\epsilon}=0
$$

Мы перешли здесь к ампутированной функции $\left\{G_{4}^{(N)}\right\}$, опустив очевидный множитель $1 /|\mathbf{p}|^{8}$. Подставляя вычисленную нами функцию $\left\{G_{4}^{(N)}\right\}$ (сумму выражений $(32)$ и $(35)$ ), а также учитывая, что

$$
C_{4}^{(N+1)} \approx-N C_{4}^{(N)}\left(1+\frac{3+n / 2}{N}\right)
$$

получаем окончательный результат для асимптотики $\left\{Z_{g}\right\}$ :

$$
\left\{Z_{g}^{(N)}\right\}_{\text {asymp }}=-\frac{C_{4}^{(N)}}{16 \pi^{2}} e^{\alpha a}\left(1+\frac{3+n / 2+\widetilde{W}_{4}-W_{4}}{N}\right) .
$$


Здесь и далее $Z_{i}^{(N)}$ в окончательных выражениях обозначает коэффициент при $u^{N}$ в выражении для $Z_{i}, u \equiv g /\left(16 \pi^{2}\right)$, как это принято в схеме МВ [11].

Получив данный результат, интересно выяснить, насколько учет поправки по $1 / N$ к асимптотике (38) улучшает аппроксимацию коэффициентов $Z_{g}^{(N)}$. В табл. 1 приведены для сравнения точные $\left(\left\{Z^{(N)}\right\}_{\text {exact }}\right)[11]$, а также асимптотические $\left(\left\{Z^{(N)}\right\}_{\text {asymp }}\right)$ значения коэффициентов в случае $n=1$ без учета и с учетом поправочного члена. Можно видеть, что учет вычисленной в данной работе поправки к асимптотической формуле Липатова приводит к сушественному улучшению результатов - последний точно вычисленный вклад $\left\{Z^{(5)}\right\}_{\text {ехасt }}$ отличается от соответствующего асимптотического значения теперь уже не в семьдесят пять, а примерно в два раза, причем наблюдается монотонность в приближении $\left\{Z^{(N)}\right\}_{\text {exact }} \mathrm{K}\left\{Z^{(N)}\right\}_{\text {asymp }}$. Аналогичная ситуация имеет место и для $n=2,3, \ldots$.

ТАБлицА 1. Точно вычисленные члены разложения констант ренормировки $\left\{Z^{(N)}\right\}_{\text {exact }}$ (однокомпонентное поле) и асимптотические значения членов разложения констант ренормировки $\left\{Z^{(N)}\right\}$ asymp

\begin{tabular}{|c|c|c|c|c|}
\hline$n=1$ & $N=2$ & $N=3$ & $N=4$ & $N=5$ \\
\hline точное значение $Z_{g}$ по ТВ & -2.833 & 10.85 & -67.90 & 569.7 \\
\hline асимпт. значение $Z_{g}$ в гл. порядке по $1 / N$ & -0.0049 & 0.062 & -0.68 & 7.51 \\
\hline асимпт. значение $Z_{g}$ с поправкой по $1 / N$ & -0.405 & 3.42 & -28.4 & 251 \\
\hline
\end{tabular}

Итоговая формула (38) позволяет также провести сравнение известных порядков $(4-\epsilon)$-разложения и соответствуюших асимптотических значений для критического индекса $\eta$. Упомянутый индекс определяется, как известно, соотношениями

$$
\eta=2 \gamma_{\varphi}\left(g_{*}\right), \quad \gamma_{i}^{(N)}{ }_{\text {asymp }}=-N\left\{Z_{i}^{(N)}\right\}_{\text {asymp }}
$$

положение фиксированной точки $g_{*}$ задается уравнением $\beta\left(g_{*}\right)=-g_{*}\left(\epsilon+\gamma_{g}\left(g_{*}\right)\right)=0$. При вычислении первой поправки по $1 / N$ в выражении для $\eta_{\text {asymp }}^{(N)}$ константу ренормировки $Z_{\varphi}^{(N)}$ достаточно учесть лишь в главном по $1 / N$ порядке; соответствуюшее выражение

$$
Z_{\varphi}^{(N)} \text { asymp }=-\frac{C_{4}^{(N)}(n+2) e^{\alpha a}}{2304 \pi^{2} N^{2}}
$$

было вычислено в $[3]^{2)}$. Окончательно для асимптотики высоких порядков разложения

\footnotetext{
2) При цитировании упомянутой формулы мы исправили опечатку в [3] (потерянный множитель $1 / N$ в первом слагаемом формулы (I.35)) и несколько упростили ответ, используя введенные в данной статье константы.
} 
индекса $\eta$ мы получили

$$
\begin{gathered}
\eta_{\text {asymp }}^{(N)}=-\frac{C_{4}^{(N)} Z_{\varphi}^{(2)}}{2 \pi^{2} a^{N+1}} e^{\alpha a+2 Z_{g}^{(2)} / a}\left[1+\frac{\Omega}{N}\right] \\
\Omega=-\widetilde{W}_{4}+W-\frac{7+n}{2}\left[e^{-2 Z_{g}^{(2)} / a}+1\right]+\frac{3 Z_{g}^{(3)}}{a}-\frac{4 Z_{g}^{(2)}}{a^{2}}+ \\
+\frac{3 Z_{g}^{(2)}}{a}+4\left(\frac{Z_{g}^{(2)}}{a}\right)^{2}-\frac{n}{2}-\frac{9 Z_{\varphi}^{(3)}}{4 Z_{\varphi}^{(2)}}+\frac{(n+2) a}{576}
\end{gathered}
$$

Входящие в это выражение начальные коэффициенты разложения констант ренормировки в $(4-\epsilon)$-схеме МВ имеют следуюший вид:

$$
\begin{gathered}
Z_{g}^{(1)} \equiv a, \quad Z_{g}^{(2)}=-\frac{3 n+14}{6}, \quad Z_{g}^{(3)}=\frac{33 n^{2}+922 n+2960+96 \zeta(3)(5 n+22)}{648} \\
Z_{\varphi}^{(2)}=-\frac{n+2}{72}, \quad Z_{\varphi}^{(3)}=\frac{(n+2)(n+8)}{1296}
\end{gathered}
$$

Как и в случае константы ренормировки $Z_{g}$, поправка к асимптотике для критического индекса $\eta$ сушественно улучшает асимптотический результат (см. табл. 2).

ТАБлицА 2. Точно вычисленные члены разложения критического индекса $\left\{\eta^{(N)}\right\}_{\text {exact }}$ (однокомпонентное поле) и асимптотические значения членов разложения критического индекса $\left\{\eta^{(N)}\right\}_{\text {asymp }}^{3 \text { ) }}$

\begin{tabular}{|c|c|c|c|c|}
\hline$n=1$ & $N=2$ & $N=3$ & $N=4$ & $N=5$ \\
\hline точное значение $\eta$ по ТВ & 0.0185 & 0.0187 & -0.00833 & 0.02566 \\
\hline асимпт. значение $\eta$ в гл. порядке & $9.2 \cdot 10^{-6}$ & $-3.9 \cdot 10^{-5}$ & $1.4 \cdot 10^{-4}$ & $-5.2 \cdot 10^{-4}$ \\
\hline асимпт. значение $\eta$ с поправкой & $-8.2 \cdot 10^{-4}$ & 0.00229 & -0.00626 & 0.01821 \\
\hline
\end{tabular}

\section{8. ЗАКЛЮЧЕНИЕ}

Мы вычислили поправку к асимптотической формуле Липатова с учетом особенностей $(4-\epsilon)$-схемы МВ. Данная поправка сушественно улучшает выход коэффищиентов ТВ на асимптотический режим: уже пятипетлевые результаты удовлетворительно описываются теперь асимптотической формулой. Таким образом, мы вынуждены заключить, что учет данной поправки необходим для улучшения точности вычислений при пересуммировании результатов методами Бореля, Бореля-Лероя.

Кроме того, мы продемонстрировали, что дальнейшее улучшение точности вычисления критических индексов может осуществляться не только при помощи вычисления

\footnotetext{
${ }^{3)}$ В таблице по сравнению с [3] исправлены опечатки.
} 
последующих порядков ТВ, но и путем расчета последующих поправок по $1 / N$ к липатовской асимптотике, причем в рамках $(4-\epsilon)$-схемы МВ значение последнего метода особенно велико. Действительно, согласно прогнозу, данному для данной схемы в работе [3], ряды $(4-\epsilon)$-разложений выйдут на главный член липатовской асимптотики лишь в десятом - одиннадцатом порядке ТВ. Поскольку количество диаграмм и, следовательно, объем вычислений лавинообразно растут с ростом порядка ТВ, не исключено, что поиск дальнейших поправок к липатовской асимптотике является перспективной задачей на данном этапе исследований.

\section{Список литературы}

[1] Л. Н. Липатов. ЖЭТФ. 1977. Т. 72. С. 411.

[2] E. Brézin, J. C. Le Guillou, J. Zinn-Justin. Phys. Rev. D. 1977. V. 15. P. 1544.

[3] М.В. Комарова, М. Ю. Налимов. ТМФ. 2001. Т. 126. С. 409.

[4] Ю.А. Кубъишин. ТМФ. 1983. Т. 57. С. 363.

[5] А.Г. Уиверидзе. Ядерная физика. 1983. Т. 38. С. 798.

[6] J. Honkonen, M. Nalimov. Phys. Lett. B. 1999. V. 459. P. 582.

[7] J. Honkonen, M. Komarova, M. Nalimov. Acta Physica Slovaca. 2002. V. 52. P. 303.

[8] И. М. Суслов. ЖЭТФ. 1997. Т. 111. С. 220.

[9] М. В. Комарова, М. Ю. Налимов. ТМФ. 2001. Т. 129. С. 387.

[10] V. G. Makhankov. Phys. Lett. A. 1977. V. 61. P. 431.

[11] A.H. Васильев. Квантовополевая ренормгруппа в теории критического поведения и стохастической динамике. С-Пб.: Изд-во ПИЯФ, 1998.

[12] A. J. McKane, D. J. Wallace. J. Phys. A. 1978. V. 11. P. 2285.

Поступила в редакцию 30.VII.2004 г. 